\title{
Nonlinear Closure Relations for Electron Transport in Hydrodynamical Models
}

\author{
A. Salhoumi ${ }^{1,2}$ \\ ${ }^{1}$ Faculté des Sciences Juridiques Economiques et Sociales Ain Sebaa, Université Hassan II-Mohammedia, Beausite, \\ BP 2634, Ain sebaa, Casablanca, Morocco \\ ${ }^{2}$ Laboratoire de Physique de la Matière Condensée (LPMC), Faculté des Sciences Ben M'sik, Université Hassan II-Mohammedia, \\ BP 7955, Casablanca, Morocco \\ Correspondence should be addressed to A. Salhoumi; ahmedsalhoumi@gmail.com
}

Received 9 September 2013; Accepted 22 October 2013

Academic Editor: Brian J. Edwards

Copyright (c) 2013 A. Salhoumi. This is an open access article distributed under the Creative Commons Attribution License, which permits unrestricted use, distribution, and reproduction in any medium, provided the original work is properly cited.

Closure relations problem of hydrodynamical models in semiconductors is considered by expressing third-and fourth-order closure relations for the moments of the distribution function in terms of second-order Lagrange multipliers using a generalized MaxwellBoltzmann distribution function within information theory. Calculation results are commented and compared with others to justify the accuracy of the approach developed in this paper. The comparison involves, in the first part with good agreements, the closure relations results obtained within extended thermodynamics which were checked by means of Monte Carlo simulations, in the second part, the results obtained by Grad's method which expands the distribution function up to fourth-order in Hermite polynomials. It is seen that the latter method cannot give any restriction on closure relations for higher-order moments, within the same conditions proposed in our approach. The important role of Lagrange multipliers for the determination of all closure relations is asserted.

\section{Introduction}

The analysis of transport in small semiconductor devices is essential for the optimization of their functioning. Such transport could in principle be described by means of Boltzmann transport equation (BTE) for charge carriers. However, in small devices the electric fields are extremely large, and therefore nonlinear effects are unavoidable $[1,2]$ which leads to insurmountable difficulties to obtain solutions. Notwithstanding this, BTE contains more information than needed in practical applications. It is common in practice to consider only the lowest-order moments of the distribution function, which are directly related to density, charge flux, kinetic energy, heat flux, and so on. These variables are measured and controlled. This kind of approach is called a hydrodynamical approach [3-5].

The basic model, in which the various steps and approximations are derived and discussed in detail, is due to Blotekjaer [4]. So as to close the set of balance equations considered by Blotekjaer, one assumes that higher-order moments have the value appropriate for a displaced Maxwellian. A slightly different model has been suggested by Hänsch and MiuraMattausch [6]. In their model, the distribution function is expanded in Legendre polynomials and only the first two terms in the expansion are retained. Only the five balance equations for particle number, momentum, and energy are considered; then the closure is accomplished by means of the Wiedemann-Franz law for heat flux. Both models $[4,6]$ are then further simplified in order to provide a manageable set of equations appropriate for the device simulation. However, for more accurate results, the full models must be registered. In approaches similar to those cited in references $[4,6]$, Woolard et al. [7] and Thoma et al. [8] have proposed models taking into account the nonparabolicity of the band structure of the crystal. All these approaches have in common the assumption that some higher-order moments can be calculated by means of a displaced Maxwellian, such an approximation is rather rough and imprecise and its range of validity needs to be assessed [9].

Another method suitable for deriving hydrodynamicallike equations is Grad's method of moments [10]. This method 
yields, with an appropriate truncation, a set of evolution equations for the thirteen fields comprising, beside the five balance laws corresponding to particle number, momentum, and energy, rate-type equations for heat flux and anisotropic stress. These equations are known to describe dilute gases only near thermal equilibrium and fail in nonequilibrium situations. Note that Banach and Larecki [11] proposed a generalized Grad's method, which begins by expanding the phase density about an anisotropic Planck function, in order to close systems of moment equations in nonequilibrium situations.

Extended irreversible thermodynamics $[1,2]$ and extended thermodynamics $[12,13]$ are relatively recent approaches to nonequilibrium thermodynamics phenomena $[14,15]$, which at variance with classical irreversible thermodynamics [16] incorporate higher-order moments (to be interpreted as flux of fluxes) in the thermodynamic state description of system. Though both approaches coincide at least up to second-order in the fluxes, we adopt in this paper extended thermodynamics in order to derive hydrodynamical equations for carrier transport in semiconductors and try to close the system by means of a generalized distribution function within information theory (IT) [17]. Furthermore, our approach deals with the higher-order moments based models which still require a lot of fine tuning and a detailed understanding of the underlying physical phenomena as mentioned by Grasser et al. [18]. Furthermore, two recent contributions based on the maximum entropy principle (MEP) and validated through numerical results are to underline at this stage; the first one concerns an exact closure obtained of the 8-moment model for silicon semiconductors [19], and, the second one is a technique for the construction of realizable 5-moment closures whose extension to a fully three-dimensional gas was not necessarily simple. In fact, the cost of the accurate numerical integration of multidimensional distribution functions required for the resynchronization step was expected to be overwhelming [20].

Some rudimentary results of our approach were published previously [21] which contain some difficulties in comparing with other works based on Monte Carlo simulations, specially [22]. Here, we revisit it completely to overcome difficulties and to provide accurate results. It will be done by assuming nonvanishing higher-order Lagrange multipliers (L-Multipliers). Such hypothesis is not the first one to this field but there is some relevant contributions in quantum regime due to Trovato and Reggiani [23-26], in which they used quantum maximum entropy principle (QMEP) and dealt with closure problems by assuming that L-Multipliers could be expanded in powers of $\hbar^{2} ; \hbar$ being the reduced Planck constant, in order to determine the reduced Wigner function [27] for equilibrium and nonequilibrium conditions. Thus, we compare and comment briefly, the aims and the main results of the recent unified physical approach [25] with those of the present paper. Finally, an attempt to solve the closure problem by means of Grad's method will show within the same conditions proposed to deal with closure relations (CR) in our approach.
The plan of this paper is as follows: in Section 2, we recall the basic formalism of the hydrodynamic description in order to write the CR, that is, third- and fourthorder moment tensors in terms of lower-order quantities. In Section 3, we summarize some foregoing works dealing with the CR problem. Section 4 presents the treatment of the closure problem, in the case of an ideal gas under heat flux or subject to both heat flux and viscous pressure by expanding a generalized distribution function up to secondorder. Furthermore, we compare our results with other works. In Section 5, we summarize for comparison some results of the unified physical approach underlined above, particularly those concerning nonlinear CR. Section 6 is devoted to concluding remarks. The detail of some expressions used in this paper and some useful integrals are included as Appendices A, B, and C.

\section{Hydrodynamic Description}

The evolution equations for the moments of the distribution function are directly obtained from the semiclassical BTE for charge carriers [3]. In the case of electrons in the conduction band of a semiconductor it is written as

$$
\frac{\partial f}{\partial t}+\mathbf{v}(\mathbf{k}) \cdot \nabla f-e \mathbf{E} \cdot \nabla_{\mathbf{k}} f=P
$$

with $f(\mathbf{x}, \mathbf{k}, t)$ the distribution function, $\mathbf{k}$ the electron momentum, $\mathbf{v}(\mathbf{k})$ the electron group velocity given byv $(\mathbf{k})=$ $\nabla_{\mathbf{k}} \varepsilon$, where $\varepsilon$ is the energy, $e$ the absolute value of electron charge, $\mathbf{E}$ the electric field, and $P$ the collision term.

In the effective mass approximation [28], the energy is given by

$$
\varepsilon(\mathbf{k})=\frac{\mathbf{k}^{2}}{2 m^{*}} \quad \text { with } \mathbf{v}(\mathbf{k})=\frac{\mathbf{k}}{m^{*}},
$$

where $m^{*}$ is the effective electron mass which in silicon is $m^{*}=0.26 m_{e}$ with $m_{e}$ the electron mass and the reduced Planck's constant is taken as $\hbar=1$ for convenience.

By multiplying (1) by several products of the components of the momentum vector $\mathbf{k}$ and integrating, we obtain a hierarchy of equations for the different moments of the distribution function. Thus, we obtain different hydrodynamical models which differ in the choice of selected variables and on the level at which the truncation is made in the hierarchy $[1,2]$. We will summarize these models in Section 3. For instance, let us write this hierarchy of equations $[9,14,15,29]$.

For consistency, the boundary of the first Brillouin zone is moved toward infinity, the particle density $n(\mathbf{x}, t)$ and the mean velocity $\mathbf{u}(\mathbf{x}, t)$ are defined, respectively, as $n(\mathbf{x}, t)=$ $\int d \mathbf{k} f(\mathbf{x}, t, \mathbf{k})$ and $\mathbf{u}(\mathbf{x}, t)=\int d \mathbf{k v}(\mathbf{k}) f(\mathbf{x}, t, \mathbf{k})$, where $\mathbf{J}=n \mathbf{u}$ defines the particle flux. Assuming as usual that $f(\mathbf{x}, t, \mathbf{k})$ vanishes sufficiently fast at infinity and integrating (1) in kspace, we obtain the particle continuity equation

$$
\frac{\partial n}{\partial t}+\nabla \cdot(n \mathbf{u})=0
$$


Multiplying (1) by $k^{i}$ and integrating yield the momentum balance equation

$$
\frac{\partial\left(n u^{i}\right)}{\partial t}+\frac{\partial \theta^{i j}}{\partial x^{j}}+\frac{n e E^{i}}{m^{*}}=P^{i}
$$

Multiplying (1) by $k^{i} k^{j}$ and integrating, we find the expression of stress equation for the traceless part of $\widehat{\theta}_{\langle i j\rangle}$

$$
\frac{\partial \widehat{\theta}_{\langle i j\rangle}}{\partial t}+\frac{\partial \widehat{\theta}_{\langle i j\rangle r}}{\partial x^{r}}+\frac{2 n e E_{\langle i u j\rangle}}{m^{*}}=P_{\langle i j\rangle} .
$$

We denote the completely symmetric and traceless part of a tensor $A_{i j}$ as

$$
A_{\langle i j\rangle}=\frac{1}{2}\left[A_{i j}+A_{j i}-\frac{2}{3} \delta_{i j} A_{k}^{k}\right] .
$$

From the trace of $\widehat{\theta}_{i j}$, we obtain the balance equation for the energy $W$, with $P_{W}$ the energy production,

$$
\frac{\partial W}{\partial t}+\nabla \cdot \mathbf{S}+n e \mathbf{E} \cdot \mathbf{u}=\mathbf{P}_{W} .
$$

The energy flux equation for $S_{i}$ is governed by the following equation, with $P_{i}^{\prime}$ as the energy-flux production:

$$
\frac{\partial S_{i}}{\partial t}+\frac{\partial S_{i j}}{\partial x^{j}}+e\left[E_{i} \widehat{\theta}_{i j}+\frac{1}{m^{*}} W E_{i}\right]=P_{i}^{\prime} .
$$

Here $S_{i j}$ denotes the flux of energy flux which is written as

$$
\begin{aligned}
S_{i j}= & W u_{i} u_{j}+2 n k_{B} T u_{i} u_{j}+\frac{1}{2} u^{2} n k_{B} T \delta_{i j} \\
& +\frac{m^{*}}{2}\left(2 u_{r} u_{j} \widehat{\theta}_{\langle i r\rangle}+2 u_{i} u_{r} \widehat{\theta}_{\langle j r\rangle}+u^{2} \widehat{\theta}_{\langle i j\rangle}\right) \\
& +u_{j} Q_{i}+u_{i} Q_{j}+u_{r} \widehat{\theta}_{i j r}+\widehat{\theta}_{i j r r},
\end{aligned}
$$

with $Q_{i}$ the heat flux. $\widehat{\theta}_{i j r}$ and $\widehat{\theta}_{i j r}$ are, respectively, the random parts of the third- and fourth-order moment tensors, (10).

\section{Closure Problem and Extended Thermodynamics}

As we have mentioned above, different hydrodynamical models have tried to close the hierarchy of equations for the different moments of the distribution function. The variables appearing in the thirteen-moment equations (3)(8) are the particle number density $n$, the momentum $n u^{i}$, the temperature $T$, the stress tensor $\widehat{\theta}_{\langle i j\rangle}$, the heat flux $Q_{i}$, and plus the random parts of the third-and fourth-order moment tensors $\widehat{\theta}_{i j r}$ and $\widehat{\theta}_{i j r r}$ given by

$$
\begin{gathered}
\widehat{\theta}_{i j r}=\frac{m^{*}}{2} \int f c_{i} c_{j} c_{r} d \mathbf{k}, \\
\widehat{\theta}_{i j r s}=\frac{m^{*}}{2} \int f c_{i} c_{j} c_{r} c_{s} d \mathbf{k},
\end{gathered}
$$

where the random component $\mathbf{c}$ of the moment vector $\mathbf{k}$ is used for convenience and which is defined by $\mathbf{k}=m^{*}(\mathbf{u}+\mathbf{c})$ with $\mathbf{u}$ the mean velocity.
So as to close the system at this level of approximation, we need explicit expressions for $\widehat{\theta}_{i j r}$ and $\widehat{\theta}_{i j r r}$. One way is based on the assumption that the distribution function could be approximated by a drifted-Maxwellian $[3,4,8,30]$. This approach implies the following closure relations for the random parts of the third-order and fourth-order moment tensors, (10),

$$
\begin{gathered}
\hat{\theta}_{i j r}=\frac{1}{5}\left(Q_{i} \delta_{j r}+Q_{j} \delta_{i r}+Q_{k} \delta_{i j}\right), \\
\widehat{\theta}_{i j r r}=\frac{5}{2} \frac{n\left(k_{B} T\right)^{2}}{m^{*}} \delta_{i j} .
\end{gathered}
$$

However, this model presents some limitations which makes a sounder analysis of the possible truncation of equations to optimize the description of the system desirable $[1,2]$. Therefore, CR related to the hierarchy of moment equations of the BTE take an essential role in building hydrodynamical models of carrier transport in semiconductors, and it deserves a thorough investigation starting from first principles $[12,13]$. This is done in framework of extended thermodynamics $[1,9,12-15,29]$. The critical assumption is that $\widehat{\theta}_{i j r}$ and $\widehat{\theta}_{i j r r}$ can be considered as functions of the lower-order moments of particle density, momentum, energy, components of the pressure tensor, and heat flux. Within the definition and the justification of the partial thermal equilibrium state, Anile and Muscato [29] derived the results (11) and (12) as $\widehat{\theta}_{\langle i j r\rangle}=0$ and $\widehat{\theta}_{i j r r}=(5 / 2)\left(\left(n\left(k_{B} T\right)^{2}\right) / m^{*}\right) \delta_{i j}$ which were obtained with the drift-Maxwellian assumption. The last closure led to a flux-limited expression for the heat flow by using the Schwartz's inequality as

$$
|Q| \leq Q_{\max }=3\left(\frac{5}{2}\right)^{1 / 2} \frac{n}{\sqrt{m^{*}}}\left(k_{B} T\right)^{3 / 2} .
$$

Let us mention that other attempts to investigate the fluxlimited phenomena are well known, for instance, in radiations hydrodynamics $[9,31,32]$, in plasma physics $[33,34]$ and in electronics $[1,2,29,35-38]$. Furthermore, Anile et al. have applied $[9,14,15,29,39,40]$ a method based on extended thermodynamics $[1,2,13]$ in order to establish expressions corresponding to third- and fourth-order moment tensors, that is, $\widehat{\theta}_{i j r}$ and $\widehat{\theta}_{i j r r}$, in the second-order of $Q_{i}$ and $\widehat{\theta}_{i j}$. They obtained the following constitutive equations up to secondorder about partial thermal equilibrium:

$$
\begin{aligned}
\widehat{\theta}_{\langle i j k\rangle}= & \frac{2}{5} \frac{m^{*}}{n k_{B} T}\left(Q_{i} \widehat{\theta}_{j k}+Q_{j} \widehat{\theta}_{k i}+Q_{k} \widehat{\theta}_{i j}\right. \\
& \left.-\frac{2}{15} Q_{l}\left(\widehat{\theta}_{\langle l i\rangle} \delta_{j k}+\widehat{\theta}_{\langle l j\rangle} \delta_{k i}+\widehat{\theta}_{\langle l k\rangle} \delta_{i j}\right)\right), \\
\widehat{\theta}_{i l l j}= & \left(\frac{5 n\left(k_{B} T\right)^{2}}{2 m^{*}}+\left(\frac{\sigma}{M k_{B} T_{L}}-\frac{2}{5 n k_{B} T}\right) Q^{2}\right) \delta_{i j} \\
& +\frac{2 \sigma}{n k_{B} T_{L}} Q_{i} Q_{j}+\frac{7 k_{B} T}{2} \widehat{\theta}_{\langle i j\rangle}+\frac{m^{*}}{n} \widehat{\theta}_{\langle i l\rangle} \widehat{\theta}_{\langle j l\rangle},
\end{aligned}
$$

where $T_{L}$ is the lattice temperature. 
In the constitutive equation (15), only the free parameter $\sigma$ can be determined by comparison with Monte Carlo results [22]. In order to check CR, (14) and (15), the authors evaluate the quantities $n, u^{i}, T, Q_{i}$, and $\widehat{\theta}_{\langle i j\rangle}$ by Monte Carlo simulations and by using a suitable fitting. Accordingly, they determine the free parameter $\sigma$ appearing in (15). In fact, this free parameter $\sigma$ is in the range $-0.15-+0.15$ and it gives a maximal error less than $7 \%$ with respect to the Monte Carlo calculations as the authors described. Furthermore, one finds that in (14), the error increases with electrical field E. Indeed, for $E \simeq 10^{4} \mathrm{~V} / \mathrm{cm}$ the error is $0.01 \%$ and for $E \approx 10^{5} \mathrm{~V} / \mathrm{cm}$, the error is $1 \%$. In (15), the error is $7 \%$ for $E \approx 10^{2} \mathrm{~V} / \mathrm{cm}$ and reduces to $2 \%$ for $E$ between $10^{3} \mathrm{~V} / \mathrm{cm}$ and $10^{4} \mathrm{~V} / \mathrm{cm}$.

Note that Romano [41] has dealt with another method based on a finite difference scheme of Scharfetter-Gummel type in order to simulate a consistent energy-transport model for electron transport in semiconductors devices. This last method is free of any fitting parameters. Otherwise, it is known that the maximum entropy moment systems of the gas-dynamical Boltzmann equation suffer from severe disadvantages which are related to the nonsolvability of an underlying maximum entropy moment problem unless restrictions on the choice of the macroscopic variables are made. Thus, Junk and Romano [42] showed that no such difficulties appeared in the semiconductor case if Kane's dispersion relation is used for the energy band of electrons. In addition, a hydrodynamic subband model for semiconductors had been formulated by closing the moment system derived from the Schrödinger-Poisson-Boltzmann equations on the basis of the MEP where explicit closure relations for the fluxes and the production terms were obtained taking into account scattering of electrons with acoustic and nonpolar optical phonons, as well as surface scattering. For this model, a suitable numerical scheme was presented together with simulations of a nanoscale silicon diode [43]. Evenly, Camiola et al. [44] proposed an expression of the entropy combines quantum effects and semiclassical transport by weighting the contribution of each subband with the square modulus of the envelope functions arising from the Schrödinger-Poisson subsystem. The simulations shown that their model was able to capture the relevant confining and transport features, and, assessed the robustness of the numerical scheme. By taking into account nonparabolic energy bands of Kane's type, Mascali and Romano [45] obtained explicit closure relations for fluxes and production terms, including scattering of electrons with acoustic and nonpolar optical phonons and surface scattering. Numerical simulations of a quantum diode showed the feasibility of their model and the importance of the nonparabolicity was assessed. Likewise, a recent unified physical approach developed by Trovato and Reggiani [2326] deal with the same CR problem in quantum scheme, we will report in Section 5 some results of this latter for a comparison asserting that the knowledge of the L-Multipliers implies the determination of all CR for the system.

\section{Information Theory and Closure Relations}

In the microscopic description, several methods (kinetic theory and information theory) coincide in the first-order in the fluxes. Here, we want to check disagreements in higher-orders (second-order for instance). We think that production terms and closure relations are good fields to do that. In this work, we consider only the latter ones. We apply IT [17] to deal with closure relations of a gas in nonequilibrium steady state subject to heat flux and viscous pressure. The treatment proposed here differs from that we have presented in the precedent section. Indeed, there are two modifications: (i) from a microscopic point of view, it is based on a second-order expansion of a generalized distribution function obtained from IT and (ii) from a macroscopic point of view, it is based on extended thermodynamics but with a slightly different interpretation of the inequalities arising from the second law of thermodynamics.

Thus, we consider a gas in a nonequilibrium steady state characterized by numerical density $n$, internal energy, or local equilibrium temperature $T$ and by a heat flux $\mathbf{Q}$ and viscous pressure $\hat{\theta}_{i j}$. The conditions to be imposed on the nonequilibrium distribution function $f_{\text {neq }}$ are

$$
\begin{gathered}
\int f_{\text {neq }} d \mathbf{k}=\int f_{\mathrm{eq}} d \mathbf{k}=n, \\
\int f_{\text {neq }} \mathbf{c} d \mathbf{k}=\int f_{\mathrm{eq}} \mathbf{c} d \mathbf{k}=0, \\
\int f_{\text {neq }}\left(\frac{1}{2} m^{*} \mathbf{c}^{2}\right) d \mathbf{k}=\int f_{\mathrm{eq}}\left(\frac{1}{2} m^{*} \mathbf{c}^{2}\right) d \mathbf{k}=\frac{3}{2} n k_{B} T, \\
\int f_{\text {neq }}\left(\frac{1}{2} m^{*} \mathbf{c}^{2}\right) c_{i} d \mathbf{k}=Q_{i}, \\
\int f_{\text {neq }} c_{\langle i c j\rangle} d \mathbf{k}=\widehat{\theta}_{\langle i j\rangle},
\end{gathered}
$$

where $f_{\text {eq }}$ is the local equilibrium distribution function corresponding to the number density $n$ and the internal energy $(3 / 2) n k_{B} T$. Since $f_{\text {neq }}$ differs from $f_{\text {eq }}$, the higherorder moments of $f_{\text {neq }}$, as for instance corresponding to nonconserved quantities, will in general differ from the corresponding moments (19) and (20) of $f_{\mathrm{eq}}[46]$.

4.1. System under Heat Flux and Flux Limiters. When we take into account the heat flux as the only dissipative source, the maximum entropy solution for the distribution function has the form

$$
f_{\text {neq }}=A \exp \left(-\beta \frac{1}{2} m^{*} \mathbf{c}^{2}-\left(\frac{1}{2} m^{*} \mathbf{c}^{2}-\frac{5}{2 \beta}\right) \gamma \cdot \mathbf{c}\right)
$$

where $\beta$ and $\gamma$ are the L-Multipliers to be determined from the constraints expressed by (18) and (19). $A$ is a normalization constant to be obtained from (16). The Lagrange multiplier corresponding to the velocity has been written as $-(5 / 2 \beta) \gamma$, a relation which, as it is known, satisfies the restriction (17). Therefore, the term $(5 / 2 \beta) \gamma \cdot \mathbf{c}$ follows from the requirement that the system is at rest; that is, $\langle\mathbf{c}\rangle=0$ [47]; it behaves near equilibrium as $(5 / 2 \beta) \mathbf{c} \approx\left(\left(5 k_{B} T\right) / 2\right)$ c. 
By using the shorthand notation $\alpha=(1 / 2) m^{*} \beta$ and $B(\mathbf{c})=\left((1 / 2) m^{*} \mathbf{c}^{2}-(5 / 2 \beta)\right)$, we expand (21) up to the secondorder in $\gamma$ as

$$
f_{\text {neq }}=A \exp \left(-\alpha \mathbf{c}^{2}\right)\left[1-B(\mathbf{c}) \gamma \cdot \mathbf{c}+\frac{1}{2} B^{2}(\mathbf{c})(\gamma \cdot \mathbf{c})^{2}\right] .
$$

Thus, if one restricts the expansion to the first-order in $\gamma$, one would obtain the Grad distribution function [10] corresponding to a steady heat flux without viscous pressure. Also this second-order expansion in $f_{\text {neq }}$ (which corresponds to a fourth-order expansion in c) is not exactly equal to the fourth-order expansion in the Grad's scheme as it will be commented below. Note finally that according to the usual definition of the entropy in terms of the velocity distribution function $f$

$$
\rho s=-k_{B} \int f \ln (f) d \mathbf{c} .
$$

We obtain, according to the restrictions imposed on $f$

$$
d s=k_{B} \beta d u+k_{B} \gamma \cdot d \mathbf{Q} \text {. }
$$

Therefore, the entropy is no longer the local equilibrium entropy $[1,2]$, but it depends also on the heat flux $\mathbf{Q}$ (through the Lagrange multiplier $\gamma$ ). Later, we will include also the viscous pressure $\widehat{\theta}_{i j}$ as a further independent quantity.

Introduction of (22) into (10) yields up to second-order in L-Multipliers corresponding to heat flux

$$
\begin{aligned}
& \widehat{\theta}_{i j k}=\frac{1}{5}\left(Q_{i} \delta_{j k}+Q_{j} \delta_{i k}+Q_{k} \delta_{i j}\right), \\
& \widehat{\theta}_{i j r r}=\frac{5 n}{2 m^{*} \beta^{2}} \delta_{i j}+C_{2 i j}(\beta, n) \gamma_{i} \gamma_{j},
\end{aligned}
$$

where $C_{2 i j}(\beta, n)=A \int \exp \left(-\beta\left(\left(m^{*} \mathbf{c}^{2}\right) / 2\right)\right)\left(B^{2}(\mathbf{c}) / 2\right) \mathbf{c}^{2} c_{i}^{2} c_{j}^{2} d \mathbf{c}$.

We conclude that relation (25) coincides with (11) and by eliminating the second-order correction term in the righthand side of (26) we find the same result as (12). Indeed, we can justify the second-order correction appearing in (26) by using a generalized distribution function.

Introduction of (22) into (16)-(19) yields the following simplified expressions:

$$
\begin{gathered}
A=\frac{n}{m^{*}}\left(\frac{\alpha}{\pi}\right)^{3 / 2}\left[1+\frac{5}{8} \frac{\gamma^{2}}{\alpha \beta^{2}}\right]^{-1}, \\
\frac{3}{2} n k_{B} T=\frac{3}{2} \frac{n}{\beta}\left[1+\frac{15}{8} \frac{\gamma^{2}}{\alpha \beta^{2}}\right]\left[1+\frac{5}{8} \frac{\gamma^{2}}{\alpha \beta^{2}}\right]^{-1}, \\
Q_{i}=-\frac{5}{2} \frac{n}{\beta^{3} m^{*}} \gamma_{i}\left[1+\frac{5}{8} \frac{\gamma^{2}}{\alpha \beta^{2}}\right]^{-1} .
\end{gathered}
$$

Then the relation between $Q_{i}$ and $\gamma_{i}$ can be written as

$$
Q_{i}=-\frac{A^{\prime} \gamma_{i}}{1+B^{\prime} \gamma_{i}^{2}}
$$

where $A^{\prime}$ and $B^{\prime}$ are functions of $m^{*}$ and $\beta$ and $n$ are obtained directly from (28) and are written as $A^{\prime}=(5 / 2)\left(n / \beta^{3} m^{*}\right)$ and $B^{\prime}=(5 / 8)\left(1 / \alpha \beta^{2}\right)$.
Thus, the expression (30) yields a flux limiter for the heat flux because of the relation

$$
Q \leq \frac{A^{\prime}}{2 \sqrt{B^{\prime}}}=\left(\frac{5}{4}\right)^{1 / 2} \frac{n}{\sqrt{m^{*}}}(\beta)^{-(3 / 2)} \approx\left(\frac{5}{4}\right)^{1 / 2} \frac{n}{\sqrt{m^{*}}}\left(k_{B} T\right)^{3 / 2} .
$$

This saturation behavior of heat flux is attained when $\gamma=\sqrt{1 / B^{\prime}}$. So as to have a more accurate expression of heat flux limiter, we should take higher-order terms in (22). Then, we conclude that within this approach, the heat flow is limited (31) which could described a flux-limited phenomena as mentioned below (13).

4.2. System Subject to Reduced Heat Flux and Viscous Pressure. The generalized distribution function obtained from IT which describes a priori the nonequilibrium steady states of a system subjected to heat flux $Q$ and viscous pressure $\widehat{\theta}_{i j}$ takes the following form:

$$
\begin{aligned}
f_{\text {neq }}=A \exp ( & -\beta \frac{1}{2} m^{*} c^{2}-\left(\frac{1}{2} m^{*} c^{2}-\frac{5}{2 \beta}\right) c_{i} \gamma_{i} \\
- & \left.\Gamma_{\langle j k\rangle}: m^{*} c_{\langle j c k\rangle}\right) .
\end{aligned}
$$

The expansion of this latter up to the second-order in the L-Multipliers, $\beta$, $\gamma_{i}$, and $\Gamma_{\langle j k\rangle}$ conjugated, respectively, to energy, heat flux, and viscous pressure, is written with a traceless symmetric tensor $\Lambda$ as

$$
\begin{aligned}
f_{\text {neq }}=A \exp \left(-\alpha \mathbf{c}^{2}\right)[1 & -B(\mathbf{c})\left(\gamma_{i} c_{i}\right)-m^{*}\left(\Gamma_{\langle j k\rangle}: \Lambda\right) c_{j} c_{k} \\
& +\frac{\gamma_{i}^{2}}{2} B^{2}(\mathbf{c}) c_{i}^{2}+\frac{m^{* 2}}{2}\left(\Gamma_{\langle j k\rangle}: \Lambda\right)^{2} c_{j}^{2} c_{k}^{2} \\
& \left.+m^{*} \gamma_{i}\left(\Gamma_{\langle j k\rangle}: \Lambda\right) B(\mathbf{c}) c_{i} c_{j} c_{k}\right],
\end{aligned}
$$

where $A$ is the normalization constant.

Denoting that the condition imposed on the distribution function to give the reduced heat flux is written as

$$
\int f_{\text {neq }} B(\mathbf{c}) c_{i} d \mathbf{k}=Q_{i}
$$

Hence, in order to incorporate nonlinear effects in the CR we propose up to the second-order in reduced heat flux $Q_{i}$ and viscous pressure tensor $\widehat{\theta}_{\langle i j\rangle}$, the following expressions for the third- and fourth-order moments:

$$
\begin{aligned}
\widehat{\theta}_{i j k}= & \frac{1}{5}\left(Q_{i} \delta_{j k}+Q_{j} \delta_{i k}+Q_{k} \delta_{i j}\right) \\
& +\widehat{C}_{1}\left(Q_{i} \widehat{\theta}_{j k}+Q_{j} \widehat{\theta}_{k i}+Q_{k} \widehat{\theta}_{i j}\right) \\
& +\widehat{C}_{2} Q_{l}\left(\widehat{\theta}_{\langle l i\rangle} \delta_{j k}+\widehat{\theta}_{\langle l j\rangle} \delta_{k i}+\widehat{\theta}_{\langle l k\rangle} \delta_{i j}\right), \\
\widehat{\theta}_{i j r r}= & \left(\frac{5 n}{2 m^{*} \beta^{2}}+\widehat{C}_{3} Q^{2}\right) \delta_{i j}+\widehat{C}_{4} Q_{i} Q_{j} \\
& +\widehat{C}_{5} \widehat{\theta}_{\langle i j\rangle}+\widehat{C}_{6} \widehat{\theta}_{\langle i l\rangle} \widehat{\theta}_{\langle j l\rangle},
\end{aligned}
$$

where $\widehat{C}_{i}(i=1, \ldots, 6)$ are coefficients to be determined. 
Note that all these coefficients are equal to zero in the simplified hypotheses which recover (11) and (12). In the following subsection, we will derive the coefficients $\widehat{C}_{i}(i=$ $1, \ldots, 6)$ within IT [17] by imposing some restrictions on $Q_{i}$, $\widehat{\theta}_{\langle i j\rangle}, \widehat{\theta}_{i j k}$, and $\widehat{\theta}_{i j r r}$.

4.2.1. Calculations of $\widehat{C}_{i}$. The first two hypotheses imposed over $Q_{i}$ and $\widehat{\theta}_{j k}$ to evaluate $\widehat{C}_{1}$ (resp. $\widehat{C}_{2}$ ) are $Q_{1} \neq 0$ and $\widehat{\theta}_{23} \neq 0$ (resp. $Q_{1} \neq 0$ and $\widehat{\theta}_{11} \neq 0$ ), and, the other components of $Q_{i}$ and $\widehat{\theta}_{i j}$ are equal to zero. After carrying out the calculations, (35) yields the expressions of $\widehat{C}_{1}$ and $\widehat{C}_{2}$ up to the secondorder in L-Multipliers

$$
\begin{aligned}
& \widehat{C}_{1}=\frac{\widehat{\theta}_{123}}{Q_{1} \widehat{\theta}_{23}}=\frac{2}{5} \frac{m^{*} \beta}{n}\left[1+\frac{5}{8} \frac{\gamma_{1}^{2}}{\alpha \beta^{2}}+\frac{1}{2} \frac{\left(\Gamma_{\langle 23\rangle}: \Lambda\right)^{2}}{\beta^{2}}\right], \\
& 3 \widehat{C}_{1}+2 \widehat{C}_{2}=\frac{5 \widehat{\theta}_{111}-3 Q_{1}}{5 Q_{1} \widehat{\theta}_{11}}=-\frac{69}{25} \frac{m^{*} \beta}{n}\left[\frac{\left(\Gamma_{\langle 11\rangle}: \Lambda\right)}{\beta}\right] \Phi,
\end{aligned}
$$

with

$$
\begin{gathered}
\Phi=\left(1-\frac{\left(\Gamma_{\langle 11\rangle}: \Lambda\right)}{\beta}+\frac{5}{8} \frac{\gamma_{1}^{2}}{\alpha^{2} \beta^{2}}+\frac{3}{2} \frac{\left(\Gamma_{\langle 11\rangle}: \Lambda\right)^{2}}{\beta^{2}}\right) \\
\times\left(\left(1-\frac{27}{5} \frac{\left(\Gamma_{\langle 11\rangle}: \Lambda\right)}{\beta}\right)\right. \\
\times\left(1-3 \frac{\left(\Gamma_{\langle 11\rangle}: \Lambda\right)}{\beta}+\frac{27}{8} \frac{\gamma_{1}^{2}}{\alpha^{2} \beta^{2}}\right. \\
\left.\left.+\frac{15}{2} \frac{\left(\Gamma_{\langle 11\rangle}: \Lambda\right)^{2}}{\beta^{2}}\right)\right)^{-1} .
\end{gathered}
$$

Note that we let $\left(\Gamma_{\langle 11\rangle}: \boldsymbol{\Lambda}\right) / \beta$ term without simplification by $\beta$ because it will play an important role as we will see later in this paper.

The second two hypotheses imposed over $Q_{i}$ and $\widehat{\theta}_{i j}$ to evaluate $\widehat{C}_{3}$ (resp. $\widehat{C}_{4}$ ) are $Q_{1} \neq 0$ (resp. $Q_{1} \neq 0$ and $Q_{2} \neq 0$ ), and, the other components of $Q_{i}$ and $\widehat{\theta}_{i j}$ are equal to zero. After integration, (36) gives the following expressions for $\widehat{C}_{3}$ and $\widehat{C}_{4}$ up to second-order in the L-Multipliers

$$
\begin{gathered}
\widehat{C}_{3}+\widehat{C}_{4}=\frac{\widehat{\theta}_{11 r r}-5 n / 2 m^{*} \beta^{2}}{Q_{1}^{2}}=\frac{166}{25} \frac{\beta}{n}\left[1+\frac{5}{8} \frac{\gamma_{1}^{2}}{\alpha \beta^{2}}\right], \\
\widehat{C}_{4}=\frac{\widehat{\theta}_{12 r r}}{Q_{1} Q_{2}}=\frac{119}{25} \frac{\beta}{n}\left[1+\frac{5}{8} \frac{\gamma_{1}^{2}+\gamma_{2}^{2}}{\alpha \beta^{2}}\right] .
\end{gathered}
$$

The third two hypotheses imposed over $Q_{i}$ and $\widehat{\theta}_{i j}$ to evaluate $\widehat{C}_{5}$ (resp. $\widehat{C}_{6}$ ) are $\widehat{\theta}_{12} \neq 0$ (resp. $\widehat{\theta}_{11} \neq 0$ ), and, the other components of $Q_{i}$ and $\widehat{\theta}_{i j}$ are equal to zero. Thus (36) yields the following expressions for $\widehat{C}_{5}$ and $\widehat{C}_{6}$ up to second-order in the L-Multipliers:

$$
\begin{gathered}
\widehat{C}_{5}=\frac{\widehat{\theta}_{12 r r}}{\widehat{\theta}_{\langle 12\rangle}}=\frac{7}{2} \frac{1}{\beta}, \\
\widehat{C}_{6}=\frac{\widehat{\theta}_{11 r r}-5 n / 2 m^{*} \beta^{2}-\widehat{C}_{5} \widehat{\theta}_{\langle 11\rangle}}{\widehat{\theta}_{\langle 11\rangle}^{2}} \\
=-\frac{21}{4} \frac{m^{*}}{n}\left(\left[1+\frac{8}{7} \frac{\left(\Gamma_{\langle 11\rangle}: \Lambda\right)}{\beta}-\frac{75}{7} \frac{\left(\Gamma_{\langle 11\rangle}: \Lambda\right)^{2}}{\beta^{2}}\right]\right. \\
\left.\times\left[1-\frac{\left(\Gamma_{\langle 11\rangle}: \Lambda\right)}{\beta}+\frac{3}{2} \frac{\left(\Gamma_{\langle 11\rangle}: \Lambda\right)^{2}}{\beta^{2}}\right]\right) \\
\times\left(\left[1-3 \frac{\left(\Gamma_{\langle 11\rangle}: \Lambda\right)}{\beta}+\frac{15}{2} \frac{\left(\Gamma_{\langle 11\rangle}: \Lambda\right)^{2}}{\beta^{2}}\right]^{2}\right)^{-1} .
\end{gathered}
$$

Here, we should underline that the coefficients $\widehat{C}_{i}(i=$ $1, \ldots, 6)$ depend, in our case, not only on $m^{*}, n$, and $T$ as in [22], but on the L-Multipliers $\beta, \gamma_{i}$, and $\Gamma_{\langle j k\rangle}$ by taking into account only the second-order in the expansion of the distribution function. Note that explicit expressions of the generalized distribution function corresponding to each hypothesis are given in Appendix A.

4.2.2. Comparison with Anile and Muscato [22]. In zeroorder in L-Multipliers [21] or in first-order in the LMultipliers with $\left(\Gamma_{\langle 11\rangle}: \Lambda\right) / \beta$ is equal to zero, the coefficients $\widehat{C}_{i}(i=1, \ldots, 6)$, that is, (37)-(43), become $\widehat{C}_{1} \approx$ $(2 / 5)\left(m^{*} \beta / n\right), \widehat{C}_{2} \approx-(1 / 5)\left(m^{*} \beta / n\right), \widehat{C}_{3} \approx(47 / 25)(\beta / n)$, $\widehat{C}_{4} \approx(119 / 25)(\beta / n), \widehat{C}_{5} \approx(7 / 2)(1 / \beta)$, and $\widehat{C}_{6} \approx$ $-(21 / 4)\left(m^{*} / n\right)$. At this stage, the expressions for CR near equilibrium state take the forms

$$
\begin{aligned}
\widehat{\theta}_{\langle i j k\rangle} \approx & \frac{2}{5} \frac{m^{*} \beta}{n}\left(Q_{i} \widehat{\theta}_{j k}+Q_{j} \widehat{\theta}_{k i}+Q_{k} \widehat{\theta}_{i j}\right. \\
& \left.-\frac{1}{2} Q_{l}\left(\widehat{\theta}_{\langle l i\rangle} \delta_{j k}+\widehat{\theta}_{\langle l j\rangle} \delta_{k i}+\widehat{\theta}_{\langle l k\rangle} \delta_{i j}\right)\right), \\
\widehat{\theta}_{i j r r} \approx & \left(\frac{5 n}{2 m^{*} \beta^{2}}+\frac{47}{25} \frac{\beta}{n} Q^{2}\right) \delta_{i j}+\frac{119}{25} \frac{\beta}{n} Q_{i} Q_{j} \\
& +\frac{7}{2} \frac{1}{\beta} \widehat{\theta}_{\langle i j\rangle}-\frac{21}{4} \frac{m^{*}}{n} \widehat{\theta}_{\langle i l\rangle} \widehat{\theta}_{\langle j l\rangle} .
\end{aligned}
$$

When we neglect the contribution of $\gamma_{i}$ and $\Gamma_{\langle j k\rangle}$, we can recover easily (14) and (15) established by Anile and Muscato [22], with a slight suitable difference concerning the definitions of $\mathbf{Q}, \widehat{\theta}_{i j}, \widehat{\theta}_{i j k}$, and $\hat{\theta}_{i j r r}$. In our opinion, the main difference is related to the use of different microscopic definitions of the heat flow and a vanishing expression of $\left(\Gamma_{\langle 11\rangle}: \Lambda\right) / \beta$. We will study later in this paper a nonvanishing expression of $\left(\Gamma_{\langle 11\rangle}: \Lambda\right) / \beta$ which will give us a solution to 
TABLE 1: Estimates of the free parameter $\sigma$, (46), by using data extracted from [22].

\begin{tabular}{|c|c|c|c|c|c|c|c|}
\hline Electron temperature: $T(\mathrm{~K})$ & 275.27 & 281.77 & 348.27 & 449.46 & 1694.33 & 3281.89 & 5123.61 \\
\hline Electric field: E (V/cm) & 1109 & 1449 & 5518 & 10768 & 41000 & 61230 & 80000 \\
\hline Free parameter: $\sigma$ & 2.594 & 2.534 & 2.050 & 1.589 & 0.421 & 0.218 & 0.139 \\
\hline
\end{tabular}

overcome the slight differences among our expressions and those established by Anile and Muscato [22].

Otherwise to evaluate CR, Anile and Muscato [22] used for the heat-flux the expression $\mathbf{Q}=(1 / 2) m^{*} c^{2} \mathbf{c}$ but in our case we have used $\mathbf{Q}=\left((1 / 2) m^{*} \mathbf{c}^{2}-(5 / 2 \beta)\right) \mathbf{c}$. Thus, the two definitions gave the same average value for $\mathbf{Q}$, as we have described above, but they yield different values for higherorder moments. The adopted definition in our calculation corresponds to what one calls the reduced heat flow, which it is used in fluctuation-dissipation theory where moments of $\mathbf{Q}$ play an important role.

Furthermore, if we simply compare (14) and (15) with those established in our approach (44) and (45), we can estimate the unknown free parameter $\sigma$. In fact, a definite value of this latter is provided by the maximum entropy approach or alternatively it may be determined by Monte Carlo simulations [1]. This is what we deal with in our approach based on IT. It is clear from (15) and (45) that the unknown parameter $\sigma$ is connected to $\widehat{C}_{3}$ (resp. $\widehat{C}_{4}$ ). It is a nondimensional free parameter and a function of the lattice temperature $T_{L}(300 \mathrm{~K})$ and the electron temperature $T$ as $\sigma \approx(57 / 25)\left(M T_{L} / n T\right)$ (resp. $\left.\sigma \approx(119 / 50)\left(T_{L} / T\right)\right)$ with $\beta \approx\left(1 / k_{B} T\right)$ in the case of partial thermal equilibrium.

So as to compare our results with those obtained in Monte Carlo simulations, we plot in Figure 1 for the same values of $T_{L}$ and $T$ versus field $\mathbf{E}$. The data of these latters (see Table 1) are extracted from [22]. Thus, we choose the second expression of $\sigma$ related to $\widehat{C}_{4}$ because the authors were silent about the expression of the parameter $M$ used in expression (15)

$$
\sigma \approx \frac{119}{50} \frac{T_{L}}{T} .
$$

As shown in Figure 1, we find that free parameter $\sigma$ which is obtained by using a fitting of Monte Carlo simulation data [22] becomes approximately constant at high field more than $60000 \mathrm{~V} / \mathrm{cm}$ and $\sigma \leq 0.139$.

In Figure 2, we plot the impact of electron temperature $T$ on the nondimensional free parameter $\sigma$. It is clear from Figure 2 that at high electron temperature $T$ is more than $3500 \mathrm{~K}$ and $\sigma \leq 0.13$. This last $\sigma$-value is obtained without using any fitting. Hence, we can use the expression (46) to evaluate the value of the electric field which could be applied in future experiences dealing with electron transport in semiconductors and to compare with Monte Carlo simulation results, vice versa to have electron temperature $T$ if we know electric field $E$.

4.2.3. Overcoming Slight Differences in CR with Anile and Muscato [22]. In order to overcome the slight difference between our results and those performed by Anile and Muscato [22], we have to take into account, in the first-order in L-Multipliers, a nonvanishing expression of $\left(\Gamma_{\langle 11\rangle}: \Lambda\right) / \beta$.

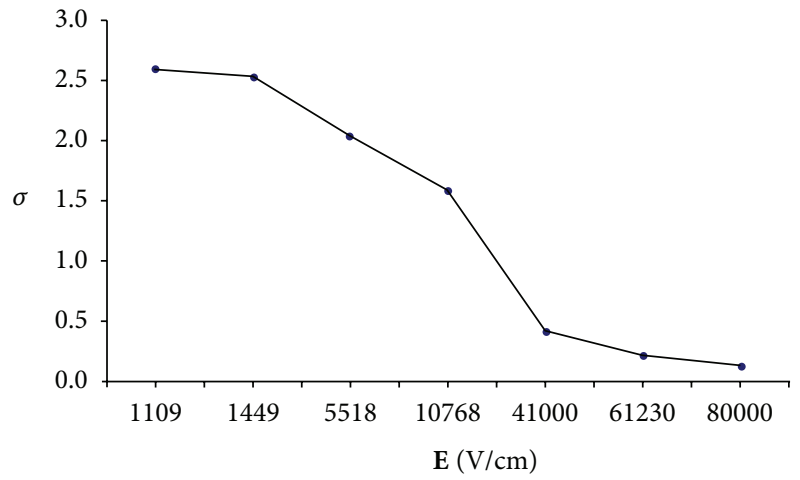

FIGURE 1: The free parameter $\sigma$ from (46) versus electric field E.

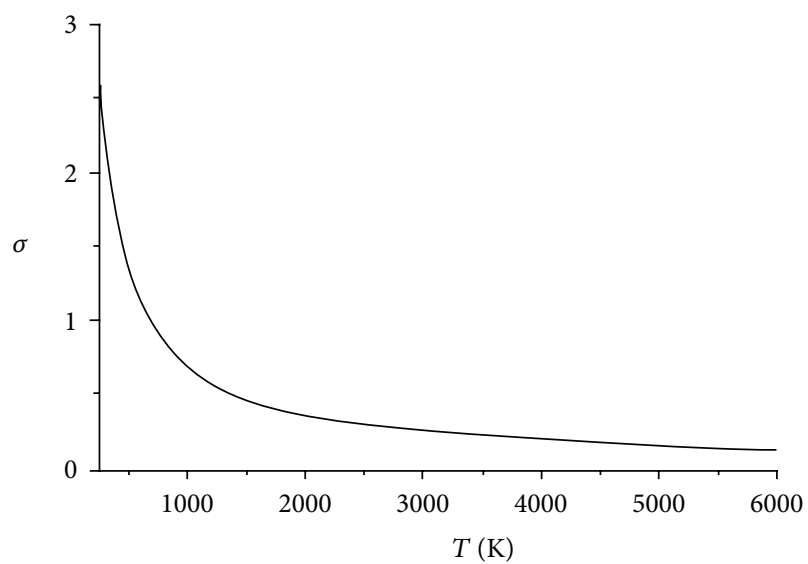

Figure 2: Determination of the free parameter $\sigma$ from (46) by varying electron temperature $T$, without using a fit nor data of Monte Carlo simulation.

Denoting firstly by $\widehat{C}_{i}^{A}(i=1, \ldots, 6)$, the coefficients related to (14) and (15) which are written as $\widehat{C}_{1}^{A} \approx(2 / 15)\left(m^{*} \beta / n\right)$, $\widehat{C}_{2}^{A} \approx-(4 / 75)\left(m^{*} \beta / n\right), \widehat{C}_{3}^{A} \approx\left(\left(\sigma / M k_{B} T_{L}\right)-(2 / 5)(\beta / n)\right)$, $\widehat{C}_{4}^{A}=\left(2 \sigma / n k_{B} T_{L}\right), \widehat{C}_{5}^{A} \approx(7 / 2)(1 / \beta)$, and $\widehat{C}_{6}^{A}=\left(m^{*} / n\right)$ with $\left(1 / k_{B} T\right) \approx \beta$. Thus, by subtracting from (38) three times (37) and adding (38) to (37), that is, (38) $-3 \times(37)$ and (38) + (37), we obtain the coefficients $\widehat{C}_{2}$ and $\widehat{C}_{1}$ in the first-order in LMultipliers with a nonvanishing $\left(\Gamma_{\langle 11\rangle}: \Lambda\right) / \beta$

$$
\begin{aligned}
\widehat{C}_{2}= & \widehat{C}_{2}^{A}\left[\frac{207}{8} \frac{\left(\Gamma_{\langle 11\rangle}: \Lambda\right)}{\beta}+\frac{45}{4}\right], \\
\widehat{C}_{1}= & -\frac{1}{5} \widehat{C}_{1}^{A}\left[\frac{207}{8} \frac{\left(\Gamma_{\langle 11\rangle}: \Lambda\right)}{\beta}+\frac{45}{4}\right] \\
& -\frac{m^{*} \beta}{n}\left[\frac{69}{100} \frac{\left(\Gamma_{\langle 11\rangle}: \Lambda\right)}{\beta}+\frac{2}{20}\right] .
\end{aligned}
$$


We find from (47) that for $\left(\Gamma_{\langle 11\rangle}: \Lambda\right) / \beta=-(82 / 207)$ we have $\widehat{C}_{2}=\widehat{C}_{2}^{A}$. This last equality into (48) yields $\widehat{C}_{1}=$ $\widehat{C}_{1}^{A}$. Thus, introducing the latter nonvanishing expression of $\left(\Gamma_{\langle 11\rangle}: \Lambda\right) / \beta$ into (43) without any trancation in LMultipliers, it gives $\widehat{C}_{6}=0.857 C_{6}^{A}$. Then, the closure relations (35) and (36) become

$$
\begin{aligned}
\widehat{\theta}_{\langle i j k\rangle} \approx & \frac{2}{5} \frac{m^{*} \beta}{n}\left(Q_{i} \widehat{\theta}_{j k}+Q_{j} \widehat{\theta}_{k i}+Q_{k} \widehat{\theta}_{i j}\right. \\
& \left.-\frac{2}{15} Q_{l}\left(\widehat{\theta}_{\langle l i\rangle} \delta_{j k}+\widehat{\theta}_{\langle l j\rangle} \delta_{k i}+\widehat{\theta}_{\langle l k\rangle} \delta_{i j}\right)\right), \\
\widehat{\theta}_{i j r r} \approx & \left(\frac{5 n}{2 m^{*} \beta^{2}}+\frac{47}{25} \frac{\beta}{n} Q^{2}\right) \delta_{i j}+\frac{119}{25} \frac{\beta}{n} Q_{i} Q_{j}+\frac{7}{2} \frac{1}{\beta} \widehat{\theta}_{\langle i j\rangle} \\
& +0.857 \frac{m^{*}}{n} \widehat{\theta}_{\langle i l\rangle} \widehat{\theta}_{\langle j l\rangle} .
\end{aligned}
$$

By comparing (49) with (14) and (15), we conclude that we have overcome the silght difference arising at zero-order and at first-order in L-Multipliers where we have used a vanishing expression of $\left(\Gamma_{\langle 11\rangle}: \boldsymbol{\Lambda}\right) / \beta$. Thus, the accuracy of our approach checked against the results of Anile and Muscato [22], involving the percent error between $\widehat{C}_{i}$ and $\widehat{C}_{i}^{A}$ is asserted such as $\widehat{C}_{1} / \widehat{C}_{1}^{A}=100 \%, \widehat{C}_{2} / \widehat{C}_{2}^{A}=100 \%$, $\widehat{C}_{5} / \widehat{C}_{5}^{A}=100 \%$, and $\widehat{C}_{6} / \widehat{C}_{6}^{A}=85.7 \%$. We recall, as we have processed above that $\widehat{C}_{4}$ and $\widehat{C}_{4}^{A}$ (resp. $\widehat{C}_{3}$ and $\widehat{C}_{3}^{A}$ ) allow us to find the nondimensional free parameter $\sigma$. It is clear now that taking into account higher-order in L-Multipliers will give better accurate results as it is seen from the calculation of coefficient $\widehat{C}_{6}$ determined without any truncation in LMultipliers.

4.2.4. Closure Relations and Grad's Method [10]. The most well-known assumed form for the distribution function is the Grad type which is based on an expansion of the distribution function in Hermite polynomials [10]. This decouples the dependence of the closure coefficients and greatly simplifies the derivation. In fact, Grad considered both 13- and 20moment closures, extensions to many moments have been considered by others $[13,48]$. However, it is an unfortunate fact that members of the Grad hierarchy suffer from several problems: (i) the distribution function is not always positive; it is therefore not a properly defined probability density function and (ii) for modest departures from local equilibrium, it is possible for the resulting moment equations to become nonhyperbolic [49-51]. Here, we assert that Grad's method fall in nonequilibrium situation. Indeed, we expand the distribution function $f_{\text {neq }}$ up to the fourth-order in the power of the velocity (this is the same order used in the precedent section). In the notation of Hermite polynomials where $H_{i}^{(n)}$ is a tensor of order $n$, Grad's distribution function writes as

$$
f_{G}=f_{\text {eq }} \sum_{n=0}^{4} \frac{1}{n !} a_{i}^{(n)} H_{i}^{(n)},
$$

where the coefficient $a_{i}^{(n)}$ is also a tensor of order $n$.
The first four closure relations are written as

$$
\begin{gathered}
\widehat{\theta}_{i j k}=\sum_{n=1}^{4} \widehat{\theta}_{i j k}^{n}, \\
\widehat{\theta}_{i j r r}=\sum_{n=1}^{4} \widehat{\theta}_{i j r r}^{n} .
\end{gathered}
$$

In order to derive the coefficients $\widehat{C}_{i}$ appearing in (35) and (36), we should use the same conditions as in Section 4.2.1; that is, $Q_{1} \neq 0$ and $\widehat{\theta}_{23} \neq 0$, and, the other components are equal to zero. Thus, up to the third-order Grad's distribution function takes the form

$$
f_{G}=f_{\mathrm{eq}}\left[1+\frac{1}{2} \frac{p_{23}}{p} c_{2} c_{3}+\frac{1}{6} \frac{Q_{1} c_{1}}{p \sqrt{R T}}\left(c^{2}-5\right)\right] .
$$

According to the last equation we observe that $\widehat{\theta}_{123}=0$, which means that $\widehat{C}_{1}=0$. Grad's distribution function can be generalized by including the full third-order contributions

$$
\begin{gathered}
f_{G}=f_{\text {eq }}\left[1+\frac{\theta_{23}}{p} c_{2} c_{3}+\frac{Q_{1} c_{1}}{p \sqrt{R T}}\left(c^{2}-5\right)\right. \\
\left.+\frac{\theta_{123}}{p \sqrt{R T}} c_{1} c_{2} c_{3}+\cdots\right],
\end{gathered}
$$

where $\widehat{\theta}_{123}$ is arbitrary.

Hence, if one imposes only conditions on $p_{23}$ and on $Q_{1}$ but not on the additional third-order terms, we observe that $\widehat{C}_{1}$ is arbitrary too, since $\widehat{C}_{1}$ is given by $\widehat{C}_{1}=\widehat{\theta}_{123} /\left(Q_{1} \widehat{\theta}_{23}\right)$. We checked also the same method for the other coefficients $\widehat{C}_{2}, \widehat{C}_{3}, \ldots, \widehat{C}_{6}$ in (35) and (36). We concluded that at higherorder, if conditions are imposed only on heat flux and viscous pressure, Grad's method cannot give any restrictions in the closure relations (35) and (36). We can understand the difference between this model and our approach based on IT. Consequently, if we compare $f_{\text {neq }}$ with $f_{G}$ used to provid $\hat{\theta}_{123}$, we will see clearly that in our approach $\widehat{\theta}_{123}$ is connected in a unique manner to $\gamma_{1}$ and $\Gamma_{\langle 23\rangle}$ but in Grad's method it is an independent coefficient. In fact, the distribution function of Grad assumes to $\widehat{\theta}_{123}=0$ in the case, where only $Q_{1} \neq 0$ and $\widehat{\theta}_{23} \neq 0$. Thus, this freedom of coefficients in Grad's method is present also for the others coefficients $\widehat{C}_{2}, \widehat{C}_{3}, \ldots, \widehat{C}_{6}$.

\section{Nonlinear Closure Relations in Quantum Scheme}

The present section is devoted to compare the aims and the results developed in Section 4 with those of the work of Trovato and Reggiani [23-26]. The authors proposed MEP in its local semiclassical and nonlocal quantum formulation as the basis of an unified physical approach. In fact, the local semiclassical case investigated MEP both in the usual kinetic theory of degenerate gases and in transport phenomena for hot carriers in nondegenerate semiconductors and mostly overviewed results obtained in the last decade. 
The nonlocal quantum case mostly summarized recent results and presented a series of original findings. Otherwise, Romano [52] proposed quantum corrections to the hydrodynamical model of semiconductors based on MEP were obtained at $\hbar^{2}$ order with a Chapman-Enskog expansion in the high field approximation, modeling the $\hbar^{2}$ part of the collision term in a relaxation form. The author deduced limiting energy-transport and drift-diffusion models.

As constraints in QMEP approach, Trovato and Reggiani [24] have determined a closed quantum hydrodynamic (QHD) system for macroscopic variables. By showing that only a higher-order expansion of the distribution function can be fruitfully applied to describe transport phenomena under conditions extremely far from thermodynamic equilibrium in the presence of very high electric fields and associated gradients such as those occurring in submicron devices. Therefore, to describe accurately the transport phenomena in submicron semiconductor devices, they have considered a nonlinear expansion of the distribution function in terms of the first thirteen relevant macrovariables involved physical interpretation. For validation purposes, the approach was applied to many one-dimensional $n^{+} n n^{+}$submicron $\mathrm{Si}$ structures by using different band structure models, different doping profiles, different lattice temperatures, and different applied biases. From the numerical results, it is shown that only a strongly nonlinear expansion provided a more accurate description of all moments [25]. We report below an abstract of those works which concern only nonlinear extended thermodynamics with thirteen moments and we comment briefly the main characteristics of this recent unified physical approach.

5.1. Unified Physical Approach due to Trovato and Reggiani [25]. In the local semiclassical case, Trovato and Reggiani [25] introduced BTE for a fermion or bose gas by developing a local theory with a temperature scheme to describe the state of a gas in which the moments of the distribution function $f(\mathbf{r}, \mathbf{u}, t)$ are taken as basic fields. Through a formal expansion around the local equilibrium configuration, they explained the extended thermodynamic theories of $N$ moments and degree $\alpha$, by determining a set of closed hydrodynamic systems for bosons and/or fermions, in the frame work of the usual gas dynamics. By introducing the kinetic field

$$
\Psi\left\{n, m u_{i}, m u^{2}, m u_{\langle i u j\rangle}, m u^{2} u_{i}\right\},
$$

where $\mathbf{u}$ denotes velocity vector; they obtained the corresponding first thirteen macroscopic variables, that is, the masse density $n$, the moment density $m u_{i}$, the energy density $m u^{2}$, the traceless momentum flux density $m u_{\langle i u j\rangle}$, and the energy flux density $m u^{2} u_{i}$ that admit a direct physical interpretation. In the same way, by defining the higherorder moments, the external field productions and collisional productions, they wrote the balance equations for usual first thirteen moments.

Thus, by considering the decomposition of a moment in its convective and central parts, they formally obtained the new set of variables $\left\{n, P, M_{\langle i j\rangle}, Q_{i}\right\}, n$ being the numerical density, $P$ the pressure, $M_{\langle i j\rangle}$ the stress deviator, and $Q_{i}$ the heat flux. Analogously, they obtained the following constitutive equations for the third- and fourth-order moments tensors $\widehat{\theta}_{i j r}$ and $\widehat{\theta}_{i l l k}$ as

$$
\begin{aligned}
\widehat{\theta}_{\langle i j k\rangle}= & M_{i j r}+3 M_{\left\langle\langle i j\rangle v_{k}\right)} \\
& -\frac{2}{5} v_{k}\left(M_{\langle r i\rangle} \delta_{j k}+M_{\langle r j\rangle} \delta_{k i}+M_{\langle r k\rangle} \delta_{i j}\right) \\
& +m n v_{(i v j v k)}, \\
\widehat{\theta}_{i l l k}= & M_{i j r}+2 v_{r} M_{\langle r i k\rangle}+7 P v_{i} v_{k}+\frac{28}{5} Q_{(i v k)} \\
& +\frac{4}{5}\left(Q_{r} v_{r}\right) \delta_{i k}+v^{2} M_{\langle i k\rangle}+P v^{2} \delta_{i k} \\
& +2 v_{r}\left(M_{\langle r i\rangle} v_{k}+M_{\langle r k\rangle} v_{i}\right)+m n v^{2} v_{i} v_{k},
\end{aligned}
$$

where $v_{i}$ is the mean velocity.

The central moments $M_{i_{1} \cdots i_{n} k}$ are expressed as

$$
M_{i_{1} \cdots i_{s} k}=\int m \widetilde{u}_{i} \cdots \widetilde{u}_{i_{s}} \widetilde{u}_{k} f(\mathbf{r}, \mathbf{u}, t) d \mathbf{u},
$$

where $\widetilde{u}=u_{i}-v_{i}$ the peculiar velocity and $s=0,1, \ldots, M$ by construction with arbitrary values for the integer $M$.

The higher-order moments $\widehat{\theta}_{\langle i j k\rangle}$ and $\widehat{\theta}_{i l l k}$ (55) are determined by decomposing the distribution function and LMultipliers into equilibrium and nonequilibrium parts where L-Multipliers are formulated versus bose and fermi integrals. In fact, by determining an analytic expression for the LMultipliers versus $n, P, Q_{i}, M_{\langle i j\rangle}$ and the fermi and bose integral functions, Trovato and Reggiani [25] obtained an explicit distribution function up to the second-order of the local equilibrium. Thus, all the results obtained for the constitutive relations are determined in terms of the fermi and/or bose functions. Therefore, only by means of an efficient evaluation of these integrals, it is possible to analyse the gas behavior for various degeneracy levels.

5.2. Comparison with Trovato and Reggiani [25]. Within the QMEP Trovato and Reggiani [25] introduced a quantum entropy functional of the reduced density matrix; the principle of quantum maximum entropy is then asserted as fundamental principle of quantum statistical mechanics. Accordingly, they developed a comprehensive theoretical formalism to construct rigorously a closed QHD transport within a Wigner function approach [25]. Their approach is formulated both in thermodynamic equilibrium and nonequilibrium conditions, and the quantum contributions are obtained by only assuming that the L-Multipliers can be expanded in powers of $\hbar^{2}$, while the classical results are recovered in the limit $\hbar \rightarrow 0$. The authors asserted that the knowledge of the L-Multipliers involves the determination of all CR for the system, up to the first quantum correction [24]. Thus, to prove the validity of the QMEP results in the case of nonlinear extended thermodynamics with thirteen moments, Trovato and Reggiani compared the analytic results existing in the literature with some recent numerical methods for the evaluation of bose and fermi integrals. 
Hence it is clear that the L-Multipliers play an important role in the conception dealing with $\mathrm{CR}$ in hydrodynamical models both in the relevant contributions of Trovato and Reggiani and in the present paper. In our case, we have assumed some restrictions on L-Multipliers where third- and fourth-order CR for the moments of the distribution function are expressed merely in terms of second-order L-Multipliers: (i) to recover the formulation of flux-limiters (30) and (ii) to overcome difficulties in comparing our results with those of Anile and Muscato [22]. In fact, we have used on the one hand a nonvanishing expression of $\left(\Gamma_{\langle 11\rangle}: \Lambda\right) / \beta$ at the first-order in L-Multipliers to find the accurate coefficients $\widehat{C}_{1}$ and $\widehat{C}_{2}$, and on the other hand the fall expression of $\widehat{C}_{6}$ without any truncation in L-Multipliers (49). Also, the fact that third- and fourth-order moments are related to the numerical values of bose and fermi integrals, in the unified physical approach due to Trovato and Reggiani, does not allow us to reach one of the main objectives of the present paper which is to find an analytical expression of the nondimensional free parameter $\sigma$ (46).

\section{Concluding Remarks}

In this paper, we have studied within extended thermodynamics implications of higher-order moments, in particular second-order moments, so as to solve the closure problem presented in hydrodynamical models. This is done by means of a generalized distribution function which is obtained from maximum-entropy argument. Thus, we have compared the results of our approach with those obtained by both models based on the assumption of a drift-Maxwellian distribution function, and, on extended thermodynamics and entropy principle; then we have found a good agreement. Further, we have applied Grad's method to solve the same nonlinear CR. In the first part, we have restricted ourself using only heat flux as dissipative source. The CR found are the same as those obtained by a drift-Maxwellian assumption of the distribution function. We have been led to an expression for the heat flux describing the flux limited phenomena occurring in the submicron-electron devices. In the second part, we have considered a generalized distribution function for a nonequilibrium system under heat flux and viscous pressure in order to obtain constitutive equations of the thirdorder and the fourth-order moments. Indeed, we have found with a slight difference similar constitutive equations as those established by Anile and Muscato [22]. This slight difference which was published previously arised from the use of a vanishing first-order in L-Multipliers. Here, it was overcome by using a nonvanishing one.

Furthermore, we conclude that considerations proposed in this paper, which concern the definition of the reduced heat flux, allow us to express the nondimensional free parameter $\sigma$ appearing in (15) as function of the lattice temperature $T_{L}$ and the electron temperature $T$. In fact, the $\sigma$-parameter appearing in fourth-order moment tensor, for partial thermal equilibrium, is related both to the Monte Carlo simulation and to a fitting as described by Anile and coworkers. Finally, we have used Grad's method to calculate the CR for higherorder moments in a simple case. We have found that this method could not give any restrictions about higher-order moments. Also, we assert as Trovato and Reggiani [2326] that the knowledge of the L-Multipliers involves the determination of all CR for the system.

In brief, we have constructed a generalized distribution function in the frame work of IT. This latter allowed us to reproduce the constitutive equations of the third-order and the fourth-order moments in terms of second-order LMultipliers and to take into account the nonlinear effects in transport-hydrodynamical models of submicron-electron devices. We should not forgot that the generalized distribution function proposed in this paper is one of several contributions dealing with the extension of the canonical distribution function by adding to the equilibrium Hamiltonian an effective Hamiltonian due to the flow $[1,2,53]$. Thus, we have considered in (21) heat flux as a supplementary constraint, and in (32) both heat flux and viscous pressure as supplementary constraints in order to conceive the generalized distribution function used in our approach. At the end, for a full exploitation of the results mentioned in the present paper involving the nonlinear CR and the maximumentropy should be tested against the Monte Carlo simulation incorporating second-order L-Multipliers.

\section{Appendices}

\section{A. Explicit Expressions of $f_{\text {neq }}$ Used in Section 4.1}

The present appendix contains the explicit expressions of the generalized distribution function corresponding to each hypothesis proposed in our paper in order to derive the coefficients $C_{i}$, that is, (37), (38), (40), (41), (42), and (43).

For the first tow hypotheses, the distribution function is written, respectively,

$$
\begin{aligned}
f_{\text {neq }}=A \exp \left(-\alpha \mathbf{c}^{2}\right)[ & 1-B(\mathbf{c}) \gamma_{1} c_{1}-m^{*}\left(\Gamma_{\langle 23\rangle}: \Lambda\right) c_{2} c_{3} \\
& +\frac{\gamma_{1}^{2}}{2} B^{2}(\mathbf{c}) c_{1}^{2}+\frac{m^{* 2}}{2}\left(\Gamma_{\langle 23\rangle}: \Lambda\right)^{2} c_{2}^{2} c_{3}^{2} \\
& \left.+m^{*} \gamma_{1}\left(\Gamma_{\langle 23\rangle}: \Lambda\right) B(\mathbf{c}) c_{1} c_{2} c_{3}\right], \\
f_{\text {neq }}=A \exp \left(-\alpha \mathbf{c}^{2}\right)\left[\begin{array}{l}
1- \\
+
\end{array}\right. & B(\mathbf{c}) \gamma_{1} c_{1}-m^{*}\left(\Gamma_{\langle 11\rangle}: \Lambda\right) c_{1}^{2} \\
& +\frac{\gamma_{1}^{2}}{2} B^{2}(\mathbf{c}) c_{1}^{2}+\frac{m^{* 2}}{2}\left(\Gamma_{\langle 11\rangle}: \Lambda\right)^{2} c_{1}^{4} \\
& \left.+m^{*} \gamma_{1}\left(\Gamma_{\langle 11\rangle}: \Lambda\right) B(\mathbf{c}) c_{1}^{3}\right] .
\end{aligned}
$$


In the conditions of the second two hypotheses, the distribution function is written, respectively,

$$
\begin{aligned}
f_{\text {neq }}=A \exp \left(-\alpha \mathbf{c}^{2}\right)[1 & -B(\mathbf{c}) \gamma_{1} c_{1}-m^{*}\left(\Gamma_{\langle 23\rangle}: \boldsymbol{\Lambda}\right) c_{2} \mathcal{c}_{3} \\
& +\frac{\gamma_{1}^{2}}{2} B^{2}(\mathbf{c}) c_{1}^{2}+\frac{m^{* 2}}{2}\left(\Gamma_{\langle 23\rangle}: \boldsymbol{\Lambda}\right)^{2} c_{2}^{2} c_{3}^{2} \\
& \left.+m^{*} \gamma_{1}\left(\Gamma_{\langle 23\rangle}: \Lambda\right) B(\mathbf{c}) c_{1} c_{2} c_{3}\right], \\
f_{\text {neq }}=A \exp \left(-\alpha \mathbf{c}^{2}\right)[1 & -B(\mathbf{c}) \gamma_{1} c_{1}-m^{*}\left(\Gamma_{\langle 11\rangle}: \Lambda\right) c_{1}^{2} \\
& +\frac{\gamma_{1}^{2}}{2} B^{2}(\mathbf{c}) c_{1}^{2}+\frac{m^{* 2}}{2}\left(\Gamma_{\langle 11\rangle}: \Lambda\right)^{2} c_{1}^{4} \\
& \left.+m^{*} \gamma_{1}\left(\Gamma_{\langle 11\rangle}: \Lambda\right) B(\mathbf{c}) c_{1}^{3}\right] .
\end{aligned}
$$

The distribution functions related to the third two hypotheses are written, respectively,

$$
\begin{array}{r}
f_{\text {neq }}=A \exp \left(-\alpha \mathbf{c}^{2}\right)\left[1-B(\mathbf{c}) \gamma_{1} c_{1}+\frac{\gamma_{1}^{2}}{2} B^{2}(\mathbf{c}) c_{1}^{2}\right], \\
f_{\text {neq }}=A \exp \left(-\alpha \mathbf{c}^{2}\right)\left[1-B(\mathbf{c}) \gamma_{1} c_{1}-B(\mathbf{c}) \gamma_{2} c_{2}\right. \\
\left.+\frac{\gamma_{1}^{2}}{2} B^{2}(\mathbf{c}) c_{1}^{2}+\frac{\gamma_{2}^{2}}{2} B^{2}(\mathbf{c}) c_{2}^{2}\right] .
\end{array}
$$

\section{B. Some Equations Used in Grad's Method}

We underline here some equations used in Grad's method to deal with CR. Indeed, the first four Hermite polynomials $H_{i}^{(n)}$ appeared in the distribution function of Grad (50) [10] may be written as

$$
\begin{gathered}
H^{(0)}=1, \\
H_{i}^{(1)}=c_{i}, \\
H_{i j}^{(2)}=c_{i} c_{j}-\delta_{i j}, \\
H_{i j k}^{(3)}=c_{i} c_{j} c_{k}-\left(c_{i} \delta_{j k}+c_{j} \delta_{i k}+c_{k} \delta_{i j}\right), \\
H_{i j k l}^{(4)}=c_{i} c_{j} c_{k} c_{l} \\
-\left(c_{i} c_{j} \delta_{k l}+c_{i} c_{k} \delta_{j l}+c_{i} c_{l} \delta_{j k}+c_{j} c_{k} \delta_{i l}+c_{i} c_{l} \delta_{i k}\right. \\
\left.+c_{k} c_{l} \delta_{i j}\right) \\
+\left(\delta_{i j} \delta_{k l}+\delta_{i k} \delta_{j l}+\delta_{i l} \delta_{j k}\right),
\end{gathered}
$$

and the coefficient tensors $a_{i}^{(n)}$ are expressed in terms of the elementary moments as

$$
\begin{gathered}
a^{(0)}=1, \\
a_{i}^{(1)}=0, \\
a_{i j}^{(2)}=\frac{p_{i j}}{p}, \\
a_{i j k}^{(3)}=\frac{s_{i j k}}{p \sqrt{R T}}, \\
a_{i j k l}^{(4)}=\frac{M_{i j k l}}{p R T}-\frac{1}{p}\left(p_{i j} \delta_{k l}+p_{i k} \delta_{j l}+p_{i l} \delta_{j k}+p_{j k} \delta_{i l}\right. \\
\left.+p_{j l} \delta_{i k}+p_{k l} \delta_{i j}\right) \\
-\left(\delta_{i j} \delta_{k l}+\delta_{i k} \delta_{j l}+\delta_{i l} \delta_{j k}\right),
\end{gathered}
$$

where $p_{m n}, s_{m n l}$, and $M_{i j k l}$ take the form

$$
\begin{gathered}
p_{i j}=\int c_{i} c_{j} f d \xi, \\
s_{i j k}=\int c_{i} c_{j} c_{k} f d \xi, \\
M_{i j k l}=\int c_{i} c_{j} c_{k} c_{l} f d \xi .
\end{gathered}
$$

Then, the simplified expressions of the closure relations (51) are given by

$$
\begin{gathered}
\widehat{\theta}_{i j k}^{(0)}=\frac{A m^{* 2}}{2} \int \exp \left(-\alpha \mathbf{c}^{2}\right) c_{i} c_{j} c_{k} d \mathbf{c}=0, \\
\widehat{\theta}_{i j k}^{(1)}=\frac{A m^{* 2}}{2} \int c_{i} c_{j} c_{k} \exp \left(-\alpha \mathbf{c}^{2}\right)\left(0 c_{m}\right) d \mathbf{c}=0,
\end{gathered}
$$

$$
\begin{gathered}
\widehat{\theta}_{i j k}^{(2)}=\frac{A m^{* 2} p^{m n}}{4 p}: \int \exp \left(-\alpha \mathbf{c}^{2}\right)\left(c_{m} c_{n}-\delta_{m n}\right) c_{i} c_{j} c_{k} c_{k} d \mathbf{c}=0 \\
\widehat{\theta}_{i j k}^{(3)}=\frac{A m^{* 2} s^{m n l}}{12 p \sqrt{R T}}: \int \exp \left(-\alpha \mathbf{c}^{2}\right) \\
\times\left(c_{m} c_{n} c_{l}-\left(c_{m} \delta_{n l}+c_{n} \delta_{m l}+c_{l} \delta_{m n}\right)\right) c_{i} c_{j} c_{k} d \mathbf{c} \\
\widehat{\theta}_{i j k}^{(4)}=0 \\
\widehat{\theta}_{i j r r}^{(0)}=\frac{m^{* 2}}{2} \int f_{\mathrm{eq}} c_{i} c_{j} \mathrm{c}^{2} d \mathbf{c} \\
\widehat{\theta}_{i j r r}^{(2)}=\frac{m^{* 2} p^{m n}}{4 p}: \int f_{\mathrm{eq}}\left(c_{m} c_{n}-\delta_{m n}\right) c_{i} c_{j} \mathbf{c}^{2} d \mathbf{c} \\
\widehat{\theta}_{i j r r}^{(4)}=\frac{m^{* 2}}{2} \frac{1}{4 !} a_{m n l r}^{(4)}: \int f_{\mathrm{eq}} H_{m n l r}^{(4)} c_{i} c_{j} \mathbf{c}^{2} d \mathbf{c} .
\end{gathered}
$$




\section{Useful Integrals}

This appendix contains some useful integrals that are needed to calculate equations presented in this paper. Let $F(\mathbf{c})$ be any scalar function of $\mathbf{c}$ such that the integrals appearing below converge, and let $c_{x}$ and $c_{y}$ be the components of the relative velocity c [1]. Then

$$
\begin{gathered}
\int F(\mathbf{c}) c_{x}^{2} d \mathbf{c}=\frac{1}{3} \int F(\mathbf{c}) \mathbf{c}^{2} d \mathbf{c}, \\
\int F(\mathbf{c}) c_{x}^{4} d \mathbf{c}=\frac{1}{5} \int F(\mathbf{c}) \mathbf{c}^{4} d \mathbf{c}, \\
\int F(\mathbf{c}) c_{x}^{2} c_{y}^{2} d \mathbf{c}=\frac{1}{15} \int F(\mathbf{c}) \mathbf{c}^{4} d \mathbf{c} .
\end{gathered}
$$

The following definite integrals are also useful:

$$
\begin{array}{r}
\int_{0}^{+\infty} \exp \left(-\alpha \mathbf{c}^{2}\right) c^{r} d \mathbf{c}=\frac{\sqrt{\pi}}{2} \frac{1}{2} \frac{3}{2} \frac{5}{2} \cdots \frac{r-1}{2} \alpha^{-(r+1) / 2} \\
r \text { even } \\
\int_{0}^{+\infty} \exp \left(-\alpha \mathbf{c}^{2}\right) c^{r} d \mathbf{c}=\frac{1}{2}\left(\frac{r-1}{2}\right) ! \alpha^{-(r+1) / 2}, \quad r \text { odd } .
\end{array}
$$

\section{Conflict of Interests}

The author declares that there is no conflict of interests in this paper.

\section{Acknowledgments}

The author thanks Professor Jou, D. from Grup de Fsica Estatistica Departament de Fsica Universitat Autonóma de Barcelona (Spain), for his precious remarks, careful reading of the paper, and helpful discussions. The author thanks also Professor Trovato, M. from Dipartimento di Matematica Università di Catania (Italy) for giving his relevant contributions [23-26] and to Professor Bennai, M. for hosting this work at LPMC. A. Salhoumi's current address is Faculté des Sciences Juridiques Economiques et Sociales Ain Sebaa, Universitxé Hassan II-Mohammedia, Ain sebaa Morocco while his hosting address is Laboratoire de Physique de la Matière Condensée (LPMC), Faculté des Sciences Ben M’sik, Université Hassan II-Mohammedia, Morocco.

\section{References}

[1] D. Jou, J. Casas-Vazquez, and G. Lebon, Extended Irreversible Thermodynamics, Springer, Berlin, Germany, 2010.

[2] G. Lebon, D. Jou, and J. Casas-Vazquez, Understanding NonEquilibrium Thermodynamics, Springer, Berlin, Germany, 2008.

[3] W. Hänsch, The Drift-Diffusion Equation and Its Application in MOSFET Modeling, Springer, Berlin, Germany, 1991.

[4] B. K. Blotekjaer, "Transport equations for electrons in twovalley semiconductors," IEEE Transactions on Electron Devices, vol. 17, no. 1, pp. 38-47, 1970.
[5] G. Baccarani and M. R. Wordeman, "An investigation on steadystate velocity overshoot in silicon," Solid State Electronics, vol. 29, pp. 970-977, 1982.

[6] W. Hänsch and M. Miura-Mattausch, "The hot-electron problem in small semiconductor devices," Journal of Applied Physics, vol. 60, no. 2, pp. 650-656, 1986.

[7] D. L. Woolard, H. Tian, R. J. Trew, M. A. Littlejohn, and K. W. Kim, "Hydrodynamic electron-transport model: nonparabolic corrections to the streaming terms," Physical Review B, vol. 44, no. 20, pp. 11119-11132, 1991.

[8] R. Thoma, A. Emunds, B. Meinerzhagen, H.-J. Peifer, and W. L. Engl, "Hydrodynamic equations for semiconductors with nonparabolic band structure," IEEE Transactions on Electron Devices, vol. 38, no. 6, pp. 1343-1353, 1991.

[9] A. M. Anile and S. Pennisi, "Thermodynamic derivation of the hydrodynamical model for charge transport in semiconductors," Physical Review B, vol. 46, no. 20, pp. 13186-13193, 1992.

[10] H. Grad, "Principles of the kinetic theory of gases in thermodynamics of gases," in Handbuck der Physik, S. Flügge, Ed., vol. 12, Springer, Berlin, Germany, 1958.

[11] Z. Banach and W. Larecki, "Nine-moment phonon hydrodynamics based on the modified Grad-type approach: formulation," Journal of Physics A, vol. 37, no. 41, pp. 9805-9829, 2004.

[12] I. Muller and T. Ruggeri, Extended Thermodynamics, Springer, New York, NY, USA, 1993.

[13] I. Muller and T. Ruggeri, Rational Extended Thermodynamics, Springer, New York, NY, USA, 1998.

[14] A. M. Anile and S. Pennisi, "Extended thermodynamics of the Blotekjaer hydrodynamical model for semiconductors," Continuum Mechanics and Thermodynamics, vol. 4, no. 3, pp. 187-197, 1992.

[15] A. M. Anile, S. Pennisi, and M. Muscato, "Extended thermodynamics of charge carrier transport in semiconductors," in Nonlinear Hyperbolic Problems: Theoretical, Applied, and Computational Aspects, A. Donato and F. Oliveri, Eds., vol. 43 of Notes on Numerical Fluid Mechanics, Vieweg, Braunschweig, Germany, 1993.

[16] S. R. de Groot and P. Mazur, Nonequilibrium Thermodynamics, Dover Publication, New York, NY, USA, 1985.

[17] R. M. Gray, Entropy and Information Theory, Springer, Berlin, Germany, 2010.

[18] T. Grasser, T. Tang, H. Kosina, and S. Selberherr, "A review of hydrodynamic and energy-transport models for semiconductor device simulation," Proceedings of the IEEE, vol. 91, no. 2, pp. 251-274, 2003.

[19] S. la Rosa, G. Mascali, and V. Romano, "Exact maximum entropy closure of the hydrodynamical model for sisemiconductors: the 8-moment case," SIAM Journal on Applied Mathematics, vol. 70, no. 3, pp. 710-734, 2009.

[20] J. G. McDonald and C. P. T. Groth, "Towards realizable hyperbolic moment closures for viscous heat-conducting gas ows based on a maximum-entropy distribution," Continuum Mechanics and Thermodynamics, vol. 25, no. 5, pp. 573-603, 2013.

[21] A. Salhoumi, M. Zakari, and Y. Boughaleb, "Nonlinear efiects in 3rd and 4th order moments of transporthydrodynamical models in semiconductors within extended thermodynamics," The Moroccan Journal of Condensed Matter, vol. 2, pp. 71-75, 1999.

[22] A. M. Anile and O. Muscato, "Extended thermodynamics tested beyond the linear regime: the case of electron transport in 
silicon semiconductors," Continuum Mechanics and Thermodynamics, vol. 8, no. 3, pp. 131-142, 1996.

[23] M. Trovato and L. Reggiani, "Quantum hydrodynamic models from a maximum entropy principle," Journal of Physics A, vol. 43, no. 10, Article ID 102001, 2010.

[24] M. Trovato and L. Reggiani, "Quantum maximum entropy principle for a system of identical particles," Physical Review E, vol. 81, no. 2, Article ID 021119, 2010.

[25] M. Trovato and L. Reggiani, "Maximum entropy principle and hydrodynamic models in statistical mechanics," Rivista del Nuovo Cimento, vol. 35, pp. 99-266, 2012.

[26] M. Trovato and L. Reggiani, "Quantum maximum entropy principle for fractional exclusion statistics," Physical Review Letters, vol. 110, no. 2, Article ID 020404, 2013.

[27] E. Wigner, "On the quantum correction for thermodynamic equilibrium," Physical Review, vol. 40, no. 5, pp. 749-759, 1932.

[28] C. Kittel, Quantum Theory of Solids, John Wiley \& Sons, New York, NY, USA, 1963.

[29] A. M. Anile and O. Muscato, "Improved hydrodynamical model for carrier transport in semiconductors," Physical Review B, vol. 51, no. 23, pp. 16728-16740, 1995.

[30] M. A. Stettler, M. A. Alam, and M. S. Lundstrom, "A critical examination of the assumptions underlying macroscopic transport equations for silicon devices," IEEE Transactions on Electron Devices, vol. 40, no. 4, pp. 733-740, 1993.

[31] A. M. Anile and V. Romano, "Covariant flux-limited diffusion theories," Astrophysical Journal Letters, vol. 386, no. 1, pp. 325329, 1992.

[32] C. D. Levermore, "Relating Eddington factors to flux limiters," Journal of Quantitative Spectroscopy and Radiative Transfer, vol. 31, no. 2, pp. 149-160, 1984.

[33] A. R. Bell, R. G. Evans, and D. J. Nicholas, "Elecron energy transport in steep temperature gradients in laser-produced plasmas," Physical Review Letters, vol. 46, no. 4, pp. 243-246, 1981.

[34] E. M. Epperlein, G. J. Rickard, and A. R. Bell, "Two-dimensional nonlocal electron transport in laser-produced plasmas," Physical Review Letters, vol. 61, no. 21, pp. 2453-2456, 1988.

[35] M. Zakari, "A continued-fraction expansion for flux limiters," Physica A, vol. 240, no. 3-4, pp. 676-684, 1997.

[36] A. Salhoumi, M. Zakari, D. Jou, J. Casa-Vazquez, and Y. Boughaleb, "A brief glimpse of extended irreversible thermodynamics theory and some of its applications," The Moroccan Journal of Condensed Matter, vol. 1, pp. 62-70, 1998.

[37] A. Salhoumi, M. Zakari, and Y. Boughaleb, "Another contribution to calculate ballistic velocity by means of continued fraction expansion within extended irreversible thermodynamics," Physics Letters A, vol. 275, no. 5-6, pp. 486-492, 2000.

[38] F. Vazquez, "Size efiects on heat transport in small systems: dynamical phase transition from difiusive to ballistic regime," Journal of Applied Physics, vol. 105, pp. 64915-64919, 2009.

[39] A. M. Anile, W. Allegretto, and C. Ringhofer, Mathematical Problems in Semiconductor Physics, vol. 1823 of Lecture Notes in Mathematics, Springer, Berlin, Germany, 2003.

[40] A. M. Anile, A. Marrocco, V. Romano, and J. M. Sellier, "2D numerical simulation of the MEP energy-transport model with a mixed finite elements scheme," Journal of Computational Electronics, vol. 4, no. 3-4, pp. 231-259, 2005.

[41] V. Romano, "2D numerical simulation of the MEP energytransport model with a finite difference scheme," Journal of Computational Physics, vol. 221, no. 2, pp. 439-468, 2007.
[42] M. Junk and V. Romano, "Maximum entropy moment system of the semiconductor Boltzmann equation using Kane's dispersion relation," Continuum Mechanics and Thermodynamics, vol. 17, no. 3, pp. 247-267, 2005.

[43] G. Mascali and V. Romano, "Numerical simulation of a hydrodynamic subband model for semiconductors based on the maximum entropy principle," Il Nuovo Cimento C, vol. 33, pp. 155-163, 2010.

[44] V. D. Camiola, G. Mascali, and V. Romano, "Numerical simulation of a double-gate MOSFET with a subband model for semiconductors based on the maximum entropy principle," Continuum Mechanics and Thermodynamics, vol. 24, pp. 417436, 2012.

[45] G. Mascali and V. Romano, "A non parabolic hydrodynamical subband model for semiconductors based on the maximum entropy principle," Mathematical and Computer Modelling, vol. 55, no. 3-4, pp. 1003-1020, 2012.

[46] J. Casas-Vázquez and D. Jou, "Nonequilibrium temperature versus local-equilibrium temperature," Physical Review E, vol. 49, no. 2, pp. 1040-1048, 1994.

[47] R. Domínguez and D. Jou, "Thermodynamic pressure in nonequilibrium gases," Physical Review E, vol. 51, no. 1, pp. 158163, 1995.

[48] H. Struchtrup, Macroscopic Transport Equations for Rareffed Gas Flows, Springer, Berlin, Germany, 2005.

[49] F. Brini, "Hyperbolicity region in extended thermodynamics with 14 moments," Continuum Mechanics and Thermodynamics, vol. 13, no. 1, pp. 1-8, 2001.

[50] M. Torrilhon, "Characteristic waves and dissipation in the 13moment-case," Continuum Mechanics and Thermodynamics, vol. 12, no. 5, pp. 289-301, 2000.

[51] M. Torrilhon, "Hyperbolic moment equations in kinetic gas theory based on multi-variate Pearson-IV-distributions," Communications in Computational Physics, vol. 7, no. 4, pp. 639-673, 2010.

[52] V. Romano, "Quantum corrections to the semiclassical hydrodynamical model of semiconductors based on the maximum entropy principle," Journal of Mathematical Physics, vol. 48, no. 12, Article ID 123504, 2007.

[53] D. Jou, J. Casas-Vázquez, and M. Criado-Sancho, "Information theory and thermodynamics of polymer solutions under flow," Physica A, vol. 262, no. 1-2, pp. 69-75, 1999. 

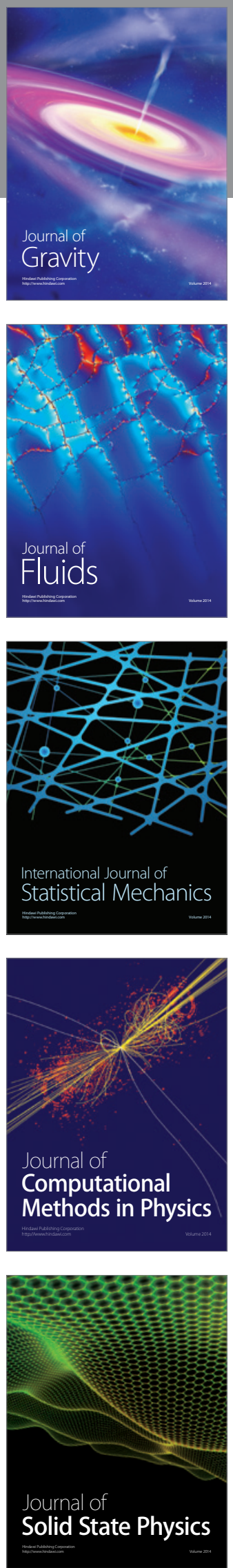

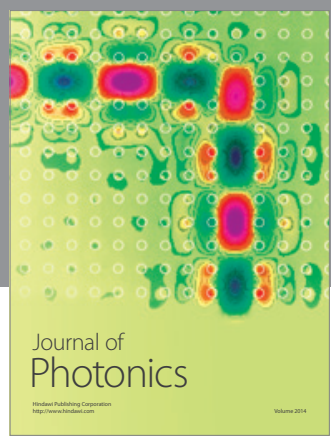

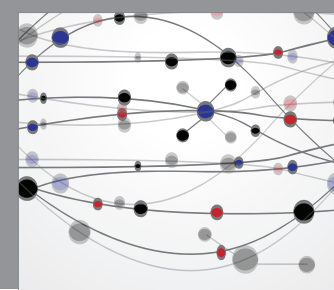

The Scientific World Journal

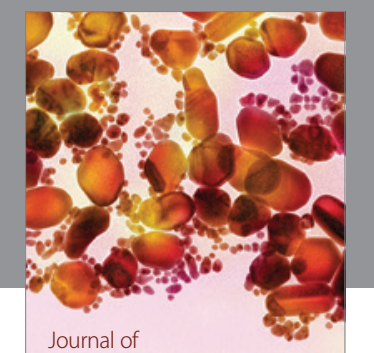

Soft Matter
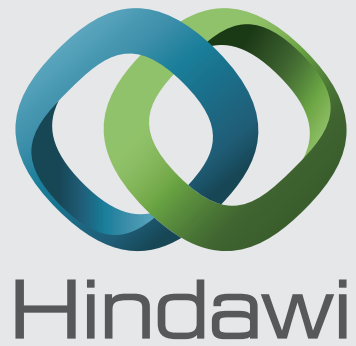

Submit your manuscripts at

http://www.hindawi.com
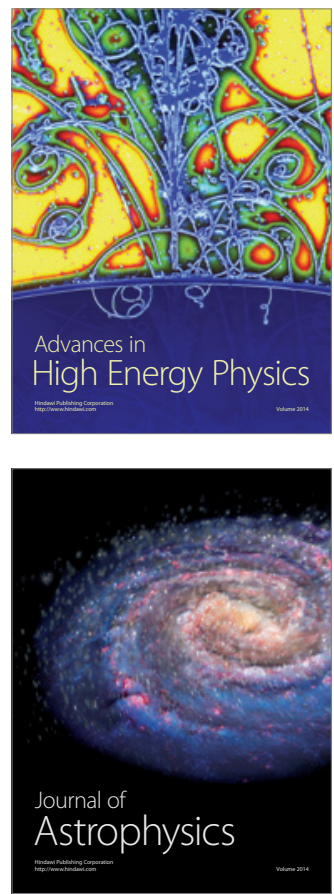
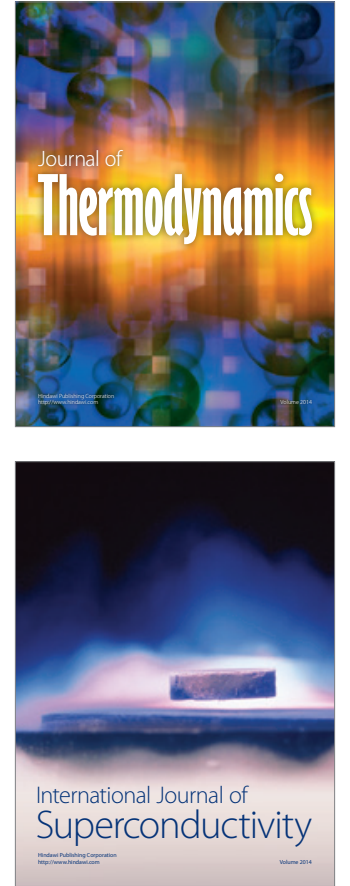
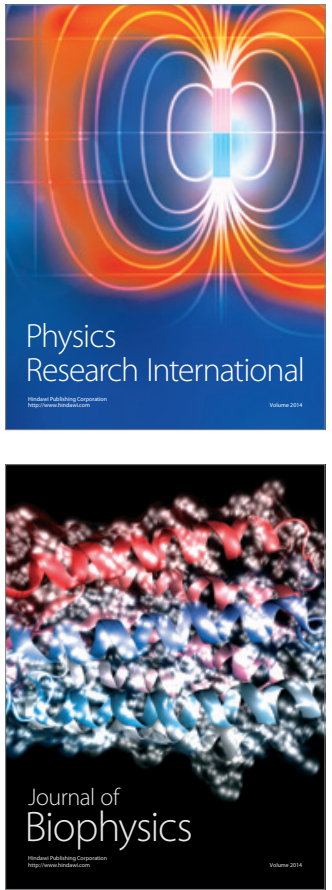
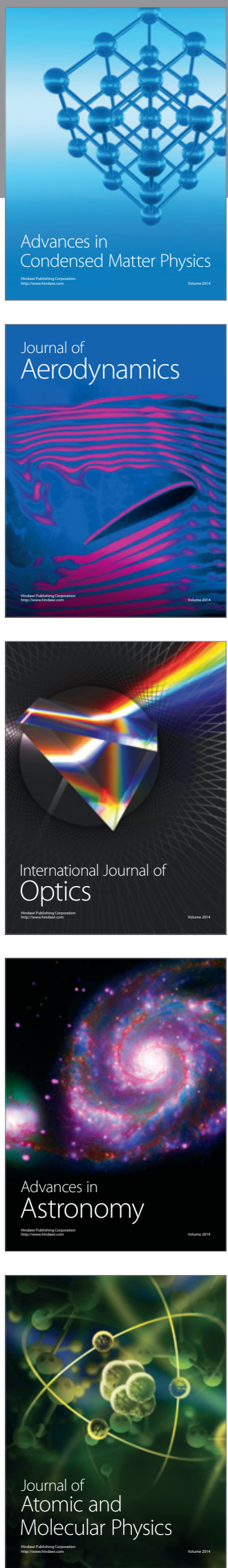